nervous system found in the present case, are the cause of the chronic chorea. We hope at some future time by the most accurate histological study in other cases, to. reach a decision as to whether any of our findings are at all frequent or even typical in this disease." W. M. L.

\title{
JACKSONIAN EPILEPSY OF SYPHILITIC ORIGIN.
}

Bernheim reports a case in the "Revue médicale de 1'Est," January I, I892. Epileptic attacks following a blow upon the head were frequent. Ten years earlier the patient, a prostitute, thirty-four years old, had been treated for syphilis; treatment renewed in form of mercurial inunction and large doses of iodide of potassium, which stopped the epileptic attacks. Two years later the patient again applied for relief of epilepsy, which had appeared after violent emotion. The relapse being due to purely psychic causes and local in its manifestation (consciousness and respiration intact), it was considered functional only, and not the result of extension of organic processes. Every cortical brain lesion being an epileptogenic centre, it can be stimulated by any dynamic influence. Attacks thus brought about may disappear spontaneously or give way to some other dynamic influence, as hypnotism, which proved efficacious in the case recorded.

L. F. B.

\section{THE ROLANDIC AREA CORTEX.}

Such is the title of a paper read before the Neurological Society of London, and contributed to the summer number of "Brain." The author, Dr. Eugene Dupiy, is well known in connection with the forcible opposition he has maintained against the conclusions drawn by the larger portion of neuro-physiologists, relative to the electrical irritability of the cortical-motor areas, and the genetic significance of the movements resulting from such stimulation. In the present article he endeavors to maintain and fortify his position. $\mathrm{He}$ states that so far no other agent than electricity produces any effect on the motor apparatus of animals when applied to the cortex. The points which, when excited by electricity give rise to a motor action, coincide with spots where arteries with nerves penetrate into the white matter or strands of fibres. He reiterates 
his theory that electrical stimulation of the cortical motor areas is manifested in motion through the action of electricity upon white matter through blood-vessels and nerves accompanying them. The fact that the experimenter is able to predict the effect to follow when a current of minimum strength applied to certain spots in the cortex of the brain of certain animals, only goes to show that certain points of the gray matter of the convolutions are situated in the lines of the least resistance. He has shown that the pia mater over the Rolandic convolution is almost a complete network of vaso-motor fibres and cells and blood-vessels which penetrate into the convolutions. One system of blood-vessels only ramifies and ends in the cortex proper, the other enters the white substance by means of larger vessels accompanied by nerves and ganglion cells. By virtue of this anatomical condition and its connections, he considers is due the motion resulting from electrical stimulation.

The criticisms of Putnam, particularly of Frank and Ferrier, he endeavors to controvert, but probably it will not be entirely satisfactory to those who have opinions on this subject. Putnam's well-known experiment to. upset Dupuy's idea that electrical irritation acts through the blood-vessels, nerves and white fibres, which consisted in slicing off the area of the cortex which is known to be stimulable, and after slicing, leaving them in situ and repeating the excitation, the same current is insufficient to call forth motor action. This Dupuy considers inconclusive on account of the physical conditions of the two experiments being different. 'The writer states, likewise, that he has shown that if the cortex is sliced off and the same minimum current used to ascertain the production of motor action be applied not directly, but after a short time, to the cut surface of the fibres, results identical with those in the first experiment are obtained, when using the same current as applied to the cortex proper before the slicing off.

Dupuy cites several experiments to prove the truth of the idea that the cortex contains centres which govern motion at all in the sense admitted by most. He cannot think it possible of a motor impulse being generated in so-called motor cells of the cortex, and traveling necessarily through and into the pyramidal tracts and cross into the cord. 'The details are too extensive to be quoted here, but they are worthy of attention.

Dupuy is very firm in his opinions that the cells of 
the cortex do not cluster into well-defined centres, either motor or sensation centres. And, likewise, that the pyramidal tracts are not the necessary connecting links between the cortex and the outgoing nerves of the cord. The substance of the article is a reiteration of the opinions previously published and known to be held by the author, which he again attempts to substantiate by logic and experiment. It is not probable, however, that very great success will attend this endeavor as the result of this most recent contribution.

J. C.

\section{SPECIAL SENSE IN EPILEPSY.}

"In Médecine Moderne," August 4, I 892, there is mention of Férés experiments in regard to special sense in epileptics, especially taste and smell. About sixty out of every hundred examined showed diminished appreciation of odors and flavors.

L. F. B.

THERAPEUTICAL.

\section{. THE TREATMENT OF EPILEPSY WITH THE BORATE OF SODA.}

Dr. Alfredo Pastena, after the employment of this drug in eight cases of epilepsy, comes to the following conclusions:

The borate of soda diminishes the number of attacks, and in many cases they are suspended for months at a time.

The best results are obtained in the classic form of epilepsy, and especially where the attacks are of long duration; also in the lighter forms, as epileptic vertigo.

The drug has no deleterious effect upon the general system, neither upon the digestive organs.

It does not act by paralyzing the motor centres, as some suppose, since in epilepsy with consecutive motor spasms, the borate of soda has little if any effect. Moreover, having administered it in mania to prove the correctness of the above-mentioned hypothesis, it gave wholly negative results. It has no marked preference for nocturnal attacks, as Stewart supposes, since patients subject to diurnal attacks have had during this treatment nocturnal attacks, and wicc virsa.

As to the dose, the author begins with 4 grammes daily, mixed with 200 grammes of water and $20 \mathrm{gr}$. of 